\title{
Persuasión moral en el marco del posconflicto en Colombia: un estudio sobre la calidad de los argumentos y la experticia de la fuente
}

\author{
Gino Marttelo Carmona Díaz; Johny Villada Zapata; Juan David Piñeres; William Jiménez Leal
}

Cómo citar este artículo:

Carmona Díaz, G.M., Villada, J., Piñeres, J. D., \& Jiménez Leal, W. (2021). Persuasión moral en el marco del posconflicto en Colombia: un estudio sobre la calidad de los argumentos y la experticia de la fuente. Acta Colombiana de Psicología, 24(2), 144-155. https://doi.org/10.14718/ACP.2021.24.2.13

Recibido, enero 12/2021; Concepto de evaluación, mayo 18/2021; Aceptado, mayo 27/2021

\author{
Gino Marttelo Carmona Díaz ${ }^{1}$ \\ ORCID: https://orcid.org/0000-0003-2621-2557 \\ Universidad de los Andes, Bogotá, Colombia. \\ Johny Villada Zapata \\ ORCID: https://orcid.org/0000-0002-9561-5344 \\ Universidad de Antioquia, Medellín, Colombia. \\ Juan David Piñeres \\ ORCID: https://orcid.org/0000-0003-1870-4113 \\ Universidad de Antioquia, Medellín, Colombia. \\ William Jiménez Leal \\ ORCID: https://orcid.org/0000-0002-8824-5269 \\ Universidad de los Andes, Bogotá, Colombia.
}

\begin{abstract}
Resumen
En el presente estudio se analiza el impacto de la calidad de los argumentos y la experticia de la fuente en la persuasión moral, así como el cambio de un juicio moral en función de un mensaje persuasivo. La investigación sobre el efecto de la calidad de los argumentos y la experticia de la fuente en la persuasión moral es escasa, a pesar de que las teorías del intuicionismo social, del proceso dual y de las convicciones morales sugieren algunos apuntes al respecto. En este trabajo, para estudiar el impacto de estos dos factores en la persuasión moral, se llevó a cabo un estudio experimental con un diseño factorial 2 (experticia de la fuente) $\times 2$ (calidad del argumento), con la participación de 433 personas. Específicamente, para evaluar el juicio moral y su eventual cambio, se construyó un dilema moral que contrapone los fundamentos morales de daño-cuidado y justicia-reciprocidad en el marco del posconflicto en Colombia. Los resultados muestran que si bien la mayoría de los participantes presentaron resistencia a la persuasión, tanto la calidad del argumento como la experticia de la fuente facilitan la persuasión, pero de forma independiente. Los resultados permiten varias reflexiones sobre las teorías del proceso dual de la persuasión y las teorías del juicio moral.

Palabras clave: calidad de los argumentos, convicciones morales, experticia de la fuente, influencia social, moralidad, persuasión, resistencia a la persuasión.
\end{abstract}

\footnotetext{
Departamento de Psicología, Cra. 1 \# 18A- 12, Edificio Franco, Bogotá, D. C., Colombia. C. P.: 111711. Tel.: $0057+133949$ 49, ext.: 2547 0057; + 301304 3082. gino.carmona@udea.edu.co; g.carmona@uniandes.edu.co

Todo el material empleado en el estudio, así como la base de datos con la información recolectada, se encuentra disponible en el portal Open Science Framework, en el siguiente enlace: https://osf.io/2nz49/

Este trabajo se llevó a cabo a partir de la investigación del autor principal para sus estudios de Maestría en Psicología de la Universidad de Antioquia.
} 


\title{
Moral persuasion in the post-conflict context in Colombia: a study on the quality of the arguments and the source expertise
}

\begin{abstract}
This study analyses the impact of argument quality and source expertise on moral persuasion, as well as the change of a moral judgment as a function of a persuasive message. Research on the effects of argument quality and source expertise on moral persuasion is scarce, although the theories of Social Intuitionism, Dual Process and Moral Convictions suggest some hints in this regard. To study the impact of these factors on moral persuasion, an experimental study was carried out with a 2 (source expertise) $\times 2$ (argument quality) factorial design with 433 participants. A particularly sensitive moral dilemma was designed to contrast the moral foundations of Harm-Care and Justice-Reciprocity in the context of the post-conflict in Colombia to evaluate moral judgment and potential change of judgment. The results show that although most of the participants presented resistance to persuasion, both the quality of the argument and the expertise of the source facilitated persuasion, albeit independently. Results also suggest several reflections on both dual process theories of persuasion and theories of moral judgement. Keywords: argument quality, moral convictions, source expertise, social influence, morality, persuasion, resistance to persuasion.
\end{abstract}

\section{Introducción}

En septiembre de 2016, Colombia se preparaba para decidir, mediante un plebiscito, si ratificaba o no los acuerdos que el gobierno nacional había logrado con el grupo insurgente FARC-EP. Si bien se anticipaba la aprobación de los acuerdos, el plebiscito estuvo precedido por un intercambio intenso de mensajes que tenían como fin persuadir a los electores de las consecuencias morales - positivas o negativas - de ratificar o no los acuerdos. Por ejemplo, a pocos días de realizarse el plebiscito, el entonces procurador General de la Nación, Alejandro Ordoñez, decía: "El acuerdo Santos-Timochenko se está utilizando como instrumento para imponer la ideología de género como norma constitucional y de esa manera dar un golpe mortal a la familia colombiana" (Revista Semana, 2016a). Finalmente, el "NO" ganó en las urnas, y el acuerdo de paz sufrió un revés significativo (Redacción El Tiempo, 2016). En los medios de comunicación se especuló que la campaña de desprestigio había logrado persuadir a los votantes de cambiar su posición mediante argumentos débiles (Revista Semana, 2016b).

Este escenario provee una oportunidad inmejorable para investigar el impacto de la calidad de los argumentos y la experticia de la fuente sobre la persuasión moral, así que, teniendo esto en cuenta, nuestro objetivo en este artículo fue explorar dicha relación a partir de un dilema moral similar al que se presentó en debates públicos sobre la ratificación de los acuerdos de paz.

Experticia de la fuente y calidad de los argumentos en la persuasión

La vasta literatura sobre la persuasión en actitudes ha mostrado que las personas pueden cambiar sus posiciones frente a un asunto tanto a través de un procesamiento esforzado y sistemático del mensaje como por medio de diferentes procesos heurísticos y automáticos (para una revisión general de los diferentes modelos de persuasión de actitudes, véase Van Lange et al., 2011). En ese sentido, se han realizado numerosos estudios para establecer qué determina que las personas cambien sus actitudes mediante un tipo de procesamiento u otro. En medio de este cuerpo de evidencia, la calidad de los argumentos y la experticia de la fuente destacan como dos de las variables más relevantes y estudiadas en los trabajos sobre persuasión (para una revisión acerca del efecto de manipulación de la experticia de la fuente, véase Pornpitakpan, 2004; para una revisión sobre el efecto de la calidad de los argumentos, véase Carpenter, 2015).

En particular, de acuerdo con los modelos duales de la persuasión, como el modelo heurístico-sistemático (MHS) (Chaiken \& Ledgerwood, 2011) y el modelo de probabilidad de elaboración (MPE) (Petty \& Briñol, 2011), el impacto de la calidad de los argumentos y la experticia de la fuente en la persuasión depende de la motivación y la capacidad para procesar el mensaje persuasivo. Así, cuando se cuenta con suficiente motivación y capacidad, el cambio de actitudes se presenta principalmente en función de la calidad de los argumentos; pero si se dispone de limitada motivación o capacidad, la persuasión se presenta especialmente por la experticia de la fuente - o cualquier otro tipo de señal heurística - (Petty \& Briñol, 2011). No obstante, desde el MPE se plantea que una variable como la experticia de la fuente puede operar sobre la persuasión de cuatro modos diferentes, además de como una señal heurística (Briñol \& Petty, 2009; Petty \& Briñol, 2006): (a) como un argumento para cambiar de actitud, (b) sesgando el procesamiento del mensaje a favor o en contra (Clark et 
146

al., 2011), (c) motivando el procesamiento esforzado del mensaje (DeBono \& Harnish, 1988), y (c) autovalidando la persuasión (Clark et al., 2011).

Ahora bien, en la revisión de literatura se encuentra que el impacto de la calidad de los argumentos y la experticia de la fuente en la persuasión han sido ampliamente documentados (Tormala \& Briñol, 2015); sin embargo, estos mismos no han sido investigados de forma sistemática respecto a los juicios morales —o persuasión moral-.

Consideramos que el dominio moral merece ser analizado de forma independiente, teniendo en cuenta que la persuasión moral es una de las formas más comúnmente empleadas para influir en los demás, especialmente en el terreno de lo político, como el caso del plebiscito colombiano lo ilustra. Adicionalmente, el cambio de los juicios morales presenta una lógica propia que es mejor comprendida por la psicología actual, y que pasamos ahora a revisar.

\section{Persuasión moral}

Si bien es escasa la literatura sobre la persuasión moral, es posible identificar tres planteamientos teóricos que se han interesado en este tema, y a partir de los cuales se pueden formular algunas hipótesis.

En primer lugar, el modelo del intuicionismo social (MIs) de Haidt (2001), que se centra en explicar los juicios morales, identifica la influencia social como un factor causal de gran relevancia. Específicamente, Haidt (2001) retoma elementos de los modelos duales de la persuasión en actitudes (Chaiken \& Ledgerwood, 2011; Petty \& Briñol, 2011) para plantear que hay dos modos de persuasión moral: (a) la persuasión social, donde los juicios morales cambian por factores sociales y elementos externos al argumento persuasivo — como la experticia de la fuente-; y (b) la persuasión razonada, en la que los juicios morales cambian por la validez de los argumentos como tal. Sin embargo, Haidt (2001) plantea que, dada la carga afectiva y la naturaleza automática de los juicios morales, los argumentos producen más persuasión por la activación de intuiciones que por la reflexión lógica que se hace de ellos. Así pues, desde esta perspectiva, la calidad de los argumentos no tiene mucha relevancia en la persuasión moral, pues lo que produce cambios en los juicios morales no es su análisis lógico, sino las intuiciones que activan. A pesar de lo anterior, Haidt $(2001,2012)$ no da cuenta de ningún estudio que trate sobre los dos modos de persuasión moral; $\mathrm{y}$, en su lugar, se apoya en el cuerpo de evidencia del MPE y el MHS, teorías que, cabe recordar, estudian especialmente la persuasión en actitudes y no en juicios morales.

Paradójicamente, la teoría de los fundamentos morales (TFM) (Graham et al., 2013), también de Haidt, ha sido más empleada para pensar la persuasión moral y política, a pesar de que no dice nada en sí misma sobre la persuasión, como sí lo hace el MIs. A partir de los trabajos que muestran que conservadores y liberales difieren en el grado de importancia que le da a unos fundamentos morales sobre otros (Graham et al., 2009), se han realizado varios estudios sobre el moral reframing (Feinberg \& Willer, 2019), es decir, la enmarcación de un mensaje en un fundamento moral relevante para una audiencia, para persuadirla sobre asuntos políticos y morales, como pueden ser el calentamiento global (Feinberg \& Willer, 2013), el matrimonio homosexual, el servicio de salud universal o el gasto militar (Feinberg \& Willer, 2015). De hecho, la TFM también se ha empleado para analizar el cambio cultural en el discurso político persuasivo (Buttrick et al., 2020) y los anuncios de las campañas presidenciales (Moses \& Gonzalez, 2015); sin embargo, ninguno de estos trabajos permite discriminar si la persuasión ocurre por una vía intuitiva o razonada.

Por otra parte, la teoría del proceso dual (TPD), de Greene (2013), que también estudia los juicios morales, señala que estos pueden ser producidos por dos tipos de procesos: uno intuitivo y otro deliberado. En esa línea, la TPD establece que la persuasión moral se puede dar tanto por una vía razonada - en la que los argumentos hacen que las personas consideren ciertos principios morales y neutralicen determinados sesgos automáticos y juicios morales intuitivos (Paxton et al., 2012) - como por una vía intuitiva —en la que determinada información produce cambios en las intuiciones de las personas, y estos cambios a su vez influyen en sus juicios morales (Paxton \& Greene, 2010) - Así pues, desde este modelo, tanto la experticia de la fuente como la calidad de los argumentos pueden impactar en la persuasión moral, por una vía u otra, dependiendo de las condiciones en las que se dé el mensaje persuasivo.

Finalmente, también se encuentra la teoría integrada de las convicciones morales (TICM), de Skitka et al. (2021), una propuesta que se centra en el estudio de las convicciones morales, es decir, aquellas actitudes que se perciben como morales. No obstante, las convicciones morales difieren de los juicios morales, pues no son una forma de cognición de primer orden en las que un espectador evalúa una situación que implica elementos morales, sino un tipo de metacognición en donde se percibe que una actitud propia presenta cierta carga moral. Un aspecto importante de la TICM es que las personas varían en el grado en que perciben que sus actitudes implican cuestiones morales (Skitka et al., 2021). Teniendo esto en cuenta, en los estudios enmarcados dentro de este modelo siempre se mide el grado en el que los participantes consideran que sus actitudes se derivan de creencias morales, en lugar de asumir que por tratar de un tema determinado ya implican una posición moral, 
como infieren los modelos que estudian los juicios morales. Adicional a esto, Skitka et al. (2021) afirman que las convicciones morales son sostenidas con independencia de la motivación de ganar el respeto de figuras de autoridad o la aceptación social, pues no dependen de preocupaciones relacionadas con fuentes externas, sino con mandatos internos que indican lo que es correcto e incorrecto. Por tanto, se puede afirmar que las personas con fuertes convicciones morales están inmunizadas contra diferentes formas de influencia social (Skitka et al., 2021).

En apoyo a esta afirmación, se ha encontrado que las personas que presentan fuertes convicciones morales rechazan y cuestionan la legitimidad de figuras de autoridad que piensen de manera diferente (Skitka et al., 2009), que se resisten a la influencia de la mayoría (Aramovich et al., 2012) y a la persuasión (Luttrell et al., 2016), y que presentan contra-conformismo - tratar de influir en las posiciones de un grupo con el cual se discrepa-(Hornsey et al., 2007). Ahora bien, aunque las convicciones morales no son lo mismo que los juicios morales, los planteamientos de este enfoque sugieren que los juicios morales son resistentes a la persuasión al margen de los argumentos o de las señales heurísticas que rodeen el mensaje; en apoyo a esta idea, se ha encontrado que identificar aspectos morales en posiciones sobre temas políticos - como la adopción homoparental o la nacionalización de la seguridad social - aumenta la resistencia a la persuasión y reduce la receptividad a otra clase de opiniones (Ben-Nun Bloom \& Levitan, 2011).

Así pues, aunque no se han realizado estudios que exploren directamente el efecto de la calidad de los argumentos y la experticia de la fuente en el contexto de la persuasión moral, los planteamientos teóricos presentados permiten inferir los efectos que podrían tener estos factores.

En concreto, a partir de los tres modelos teóricos revisados se pueden esperar resultados diferentes: primero, desde la teoría del MIS se esperaría que los juicios morales cambien por aspectos sociales - como la experticia de la fuente-, así como que la calidad de los argumentos tenga un efecto menor dada la naturaleza automática de los juicios; segundo, desde la TPD se esperaría que los juicios morales cambien tanto por procesos automáticos - como los implicados en la persuasión por la experticia de la fuente - como por procesos controlados — donde la calidad de los argumentos determina la persuasión-; y tercero, desde la TICM se esperaría que las personas que perciban sus juicios como morales se resistan a cambiar sus posiciones ante cualquier tipo de mensaje.

Los dos primeros planteamientos coinciden en que puede haber persuasión, aunque difieren en los procesos subyacentes que puedan estar implicados; y el último planteamiento, por el contrario, sugiere que la persuasión moral es mínima si las personas identifican cuestiones morales en sus juicios.

Más allá del interés teórico que tiene en sí mismo entender el mecanismo que subyace al papel de estos factores en la persuasión, es clave anticipar qué tanto influye su manipulación en casos de decisiones políticas reales, sobre todo en un momento en el que nuestras sociedades se enfrentan al reto de la desinformación sistemática (Bennett \& Livingston, 2018). En atención a esto, en el presente trabajo se estudió la influencia de la calidad de los argumentos, la experticia de la fuente y las convicciones morales en la persuasión moral, a partir de un dilema moral ambientado en el marco del proceso de paz en Colombia. A partir de los modelos teóricos revisados, es posible proponer dos hipótesis distintas: por una parte, conforme al MIS, y en contra de la TPD, se esperaría un efecto significativamente mayor de la variable independiente experticia de la fuente, comparada con la variable independiente calidad de los argumentos (H1); y, por otra, de acuerdo con la TICM, se esperaría que las personas se resistan a cambiar sus juicios morales ante cualquier tipo de mensaje, en la medida en que perciban que sus juicios se derivan de creencias morales (H2).

\section{Método}

\section{Tipo de estudio y diseño}

Se diseñó un estudio de tipo factorial 2 (argumento fuerte y argumento débil) $\times 2$ (fuente experta y fuente inexperta) entre sujetos, con la persuasión moral como variable dependiente.

\section{Participantes}

En total, participaron 448 colombianos de entre 18 y 66 años $($ Medad $=28.26, S D=9.10)$, el $66.73 \%$ con título de educación superior. Se eliminaron los datos de 15 personas, 12 por ser menores de edad y 3 que dijeron no entender el dilema, para una muestra final de 433 participantes.

\section{Procedimiento}

Los participantes accedieron a la muestra de la investigación tras ingresar a una publicación en Facebook o Twitter en la que se les invitaba a participar de un sondeo acerca de los dilemas morales en el posconflicto. La publicación los conducía a un formulario de Google donde se implementó el estudio (véase Anexo 1), y, antes de que respondieran al formulario, leyeron y aceptaron el consentimiento informado, el cual se construyó según los lineamientos del Colegio Colombiano de Psicólogos, y en el que se informaba acerca del riesgo mínimo de participar 
en el estudio, el propósito de la investigación y los usos que se le daría a la información. También, se les notificó a los participantes que con el envío del formulario autorizaban el uso de la información registrada.

En un primer momento, los participantes diligenciaron su información demográfica básica, luego se les presentó un dilema moral ambientado en el marco del posconflicto en Colombia, y más adelante se les consultó por sus juicios y convicciones morales al respecto. En este punto, se llevó a cabo la manipulación de las variables independientes: se presentó el perfil de la fuente - experta o inexperta, dependiendo de la condición - y el mensaje persuasivo - un argumento fuerte o débil, de acuerdo con la condición-. Cabe mencionar que el mensaje siempre fue contraactitudinal, de manera que se proporcionó un argumento a favor o en contra de la acción juzgada, dependiendo de si el juicio moral premensaje fue favorable o desfavorable a la acción. Después, se tomó una medida de la percepción de la calidad del argumento persuasivo y de la experticia de la fuente que lo proporcionó, con el fin de establecer si la manipulación de las variables independientes había sido exitosa. Finalmente, se volvió a consultar a los participantes por sus juicios morales frente al dilema.

\section{Medición de persuasión moral}

Para obtener información de la persuasión moral fue necesario evaluar los juicios morales antes y después de la presentación del mensaje persuasivo, lo cual se realizó por medio de un dilema moral que construido específicamente para este estudio a partir de los criterios explicitados por Christensen y Gomila (2012) en su revisión.

En particular, en el dilema se describe la actuación de una persona involucrada en el conflicto armado en Colombia, cuyas acciones reflejan una tensión entre los fundamentos morales de justicia-reciprocidad y daño-cuidado (Graham et al., 2013). El personaje principal es un personero municipal que debe decidir entre delatar a un desmovilizado del proceso de paz que omitió detalles en su declaración de la verdad, o guardar silencio teniendo en cuenta los actos de reparación que ha realizado y las implicaciones negativas que tendría para la familia del desmovilizado una posible condena por su declaración incompleta. Finalmente, el personero opta por callar frente a la declaración incompleta del desmovilizado (véase Anexo 2). Consideramos que la tensión de esta historia refleja adecuadamente el tipo de dilema al que se vieron expuestos los colombianos en los meses antes del plebiscito.

Tras leer el dilema, se solicitó a los participantes registrar su juicio moral acerca de la decisión del personero en una escala de tres ítems tipo Likert de 1 a 6: "actuó mal/actuó bien"; "inaceptable/aceptable", y "peor acción posible/mejor acción posible". Después de la presentación del mensaje persuasivo se volvió a presentar estos mismos ítems para indagar por los juicios morales posmensaje.

El mensaje persuasivo siempre fue contraactitudinal: los participantes que dieron un juicio moral reprobatorio en el premensaje recibieron un argumento a favor de la decisión del personero, mientras que quienes dieron un juicio moral aprobatorio en el premensaje recibieron un argumento en contra de la decisión del personero. De esta manera, si alguien recibió un argumento a favor, el nivel de persuasión consiste en la disminución de la reprobación, mientras que, si alguien recibió un argumento en contra, la medida de persuasión consiste en la disminución de la aprobación. Para analizar en conjunto los datos de ambos casos, se invirtieron los datos de quienes recibieron argumentos a favor. Con esta inversión siempre hubo más persuasión cuanto más hubiera disminuido la magnitud en la respuesta en el juicio posmensaje con respecto al juicio premensaje.

Por otra parte, es importante mencionar que la medida de la persuasión moral se obtuvo de estas evaluaciones a partir del cálculo de los índices de persuasión, correspondientes a la magnitud del cambio de las respuestas de los participantes en las escalas posmensaje con respecto a las escalas premensaje. Estos índices son iguales a la resta de los juicios morales posmensaje a los juicios morales premensaje. Para los resultados, los índices por encima de cero significan que hubo persuasión, los índices iguales a cero significan que hubo resistencia a la persuasión, $\mathrm{y}$, finalmente, los índices negativos significan que hubo efecto boomerang —es decir, que el juicio se polarizó-. Finalmente, se calculó un índice de persuasión por cada uno de los tres ítems, y luego del promedio de los tres índices, con el cual se realizó el análisis de los datos.

\section{Manipulación de variables independientes}

\section{Calidad de los argumentos}

Teniendo en cuenta las limitaciones de las estrategias que se han empleado para manipular la calidad de los argumentos (para una revisión sobre el tema, véase O'Keefe \& Jackson, 1995), se optó por hacer esta manipulación con base en el criterio de la relación entre las premisas y la conclusión. En ese sentido, en los argumentos fuertes se ofrecieron dos premisas relacionadas lógicamente entre ellas y de las cuales se desprendía necesariamente la conclusión; mientras que en los argumentos débiles se ofrecieron dos premisas que no estaban relacionadas y de las cuales no era posible inferir la conclusión. Cuatro expertos en derecho evaluaron los argumentos para corroborar que presentaran 
el grado de calidad deseada. Específicamente, se emplearon cuatro argumentos: fuerte a favor, débil a favor, fuerte en contra y débil en contra de la decisión del personero. La conclusión de los argumentos a favor planteaba que la decisión del personero fue moralmente correcta, mientras que la conclusión de los argumentos en contra defendía que el proceder del personero fue moralmente incorrecto (véase Anexo 2).

Para establecer qué argumento recibía cada participante se tuvo en cuenta la condición a la que fue asignado y la respuesta que dio al ítem "actuó mal/actuó bien" premensaje. Las personas que dieron en este ítem una respuesta de aprobación —respuestas 4, 5 y 6 en la escala de Likertrecibieron un argumento en contra, mientras que quienes dieron una respuesta de reprobación - respuestas 1, 2 y 3 en la escala Likert - recibieron un argumento a favor.

\section{Experticia de la fuente}

Conforme a varios de los trabajos revisados por Pornpitakpan (2004), la manipulación de esta variable se hizo a través de un texto que describía el perfil profesional de la fuente y su relación con el tema en cuestión (véase Anexo 2).

\section{Otras variables evaluadas}

\section{Eficacia en la manipulación de las variables independientes}

Con el objetivo de evaluar la eficacia en la manipulación de las variables independientes, se aplicó una escala tipo Likert de seis puntos para valorar la percepción de los participantes frente a la experticia de la fuente y la calidad del argumento recibido. Así, para evaluar la eficacia de la manipulación de la experticia de la fuente se indagó por la experticia, la experiencia y el dominio del tema de la fuente conforme a la descripción de su perfil; mientras que para evaluar la eficacia en la manipulación de la calidad de los argumentos se preguntó qué tan fuertes y convincentes fueron los argumentos presentados.

\section{Convicciones morales}

Con el fin de indagar por las convicciones morales, se incluyeron dos de las preguntas que suelen emplearse en la TICM (Skitka et al., 2021) para evaluar la investidura moral percibida en las actitudes: “¿En qué grado considera que la opinión que tiene usted sobre el tema se desprende de sus creencias sobre lo que está bien y mal?” y “¿En qué grado considera que la opinión que tiene usted sobre el tema refleja sus creencias y convicciones morales?". Las dos preguntas se hicieron en escalas tipo Likert de seis puntos.

\section{Datos sociodemográficos}

Se preguntó por la edad y el nivel educativo de los participantes, y si consideraban o no que habían sido víctimas del conflicto armado.

\section{Resultados}

En primer lugar, se evaluó la manipulación de las variables independientes, para lo cual se llevaron a cabo varias pruebas $t$ de Student con el fin de comprobar la eficacia en la manipulación de las variables independientes. Teniendo en cuenta el promedio de los tres ítems que evalúan la experticia percibida de la fuente, es posible afirmar que los participantes que recibieron el mensaje de la fuente experta la percibieron como más experta $(M=5.00 ; S D=0.06)$ que los participantes que recibieron el mensaje de la fuente inexperta $(M=2.61, S D=0.06 ; t(4.31)=-26.94, p<.001$, IC $95 \%$ diferencia de la media $[-2.56,-2.21])$. Con respecto a la calidad del argumento, a partir del promedio de los dos ítems que evalúan la calidad percibida del argumento se observa que quienes recibieron el argumento fuerte percibieron el mensaje como más fuerte y convincente $(M=4.03, S D=0.09)$ que quienes recibieron el argumento débil $(M=2.57, S D=0.09 ; t(4.31)=-10.66, p<.001$, IC $95 \%$ de la diferencia de la media $[-1.72,-1.18])$. Por lo tanto, se puede decir que la manipulación de las variables independientes fue exitosa.

En segundo lugar, se calculó el alfa de Cronbach de las escalas empleadas para evaluar el índice de persuasión. Los tres ítems empleados para evaluar el juicio moral dieron un alfa de .923 en la escala premensaje y de .929 en la escala posmensaje, lo cual da cuenta de su fiabilidad.

El análisis central consistió en una prueba ANOva con la calidad del argumento y la experticia de la fuente como variables independientes, y el promedio de los tres índices de persuasión como variable dependiente. El modelo permitió identificar un efecto principal de la calidad del argumento $\left(F(1,429)=38.039, p<.001, \eta^{2}=.077\right.$, IC $95 \%[0.038$, $0.133])$, de manera que hubo más persuasión cuando los argumentos fueron fuertes $(M=0.506, S D=0.047)$ que cuando fueron débiles $(M=0.102, S D=0.46)$; con lo cual se hace posible rechazar la primera hipótesis (H1). También, se halló un efecto principal de la experticia de la fuente $\left(F(1,429)=19.725, p<.001, \eta^{2}=.040\right.$, IC $95 \%$ $[0.013,0.086])$, según el cual se presentó más persuasión cuando la fuente era experta $(M=0.451, S D=0.046)$ que cuando era inexperta $(M=0.157, S D=0.47)$. En ambos efectos, los intervalos de confianza son consistentes con la presencia de tamaños de efectos muy cercanos a cero. Finalmente, la calidad del argumento y la experticia de la 
150

fuente no presentaron una interacción significativa $(F(1$, $429)=3.423, p>.05, \eta^{2}=.008$, IC $\left.95 \%[0.0,0.032]\right)$.

Un análisis de sensibilidad por medio del software $\mathrm{G}^{*}$ power (Faul et al., 2009) reveló que, asumiendo un poder del $96 \%$, con un alfa de $5 \%$ y el tamaño de muestra de 433 participantes, el estudio tiene un mínimo efecto detectable $\operatorname{de} f=0.21\left(\eta^{2}=0.044\right)$.

Los resultados se resumen en la Figura 1, donde se puede apreciar que la condición "argumento fuerte/fuente experta" presenta un promedio del índice de persuasión mayor al resto de las condiciones. Asimismo, se observa la gran diferencia que hay entre las condiciones de argumento fuerte y argumento débil, así como el incremento que la fuente experta da al argumento fuerte.

A pesar de estos efectos, en términos generales se halló poca persuasión, pues la mayoría de los participantes mantuvo los juicios morales expresados en el juicio moral premensaje. Por otra parte, en la Figura 2 se puede observar el porcentaje de participantes que presentaron efecto boomerang, no persuasión, o persuasión, en cada una de las condiciones para los tres ítems; donde se encuentra que la mayoría de las personas de todas las condiciones no presentó persuasión. Sin embargo, en este caso, la resistencia a la persuasión no es explicada por las convicciones morales — como se esperaría desde la TICM-. Una regresión lineal ejecutada con el promedio de los dos ítems con los que se evaluaron las convicciones morales muestra que esta variable no está relacionada de ninguna manera con la persuasión moral $(B=-0.001, B S D=0.026, \beta=-.000$, $\left.p>.05, R^{2}=-0.002\right)$.

Por otra parte, se ejecutó una prueba ANOVA factorial mixto que incluyó la calidad del argumento y la experticia de la fuente como factores fijos, y las convicciones morales como factor aleatorio. En ella, no se hallaron efectos ni interacciones significativas relacionadas con las convicciones morales. Estos dos análisis permiten rechazar la segunda hipótesis (H2).

Finalmente, se llevaron a cabo varias pruebas ANOva factorial mixto en las que se ingresaron las variables de condición de víctima, la edad y el nivel educativo como factores aleatorios. En ninguno de los modelos obtenidos se halló un efecto principal o una interacción significativa entre las variables.

\section{Discusión}

Los resultados del estudio muestran que la mayoría de los participantes conservaron sus juicios morales después de la presentación del mensaje persuasivo, la calidad de los argumentos y la experticia de la fuente presentaron un efecto principal en la persuasión moral; no obstante, no se halló una interacción entre estas dos variables. Pese a que los participantes presentaron resistencia a la persuasión, como se esperaría desde la TICM, las convicciones morales no estuvieron relacionadas con este aspecto. En lo que sigue del texto se dará una posible interpretación a estos resultados.

Figura 1.

Diagrama del perfil del modelo

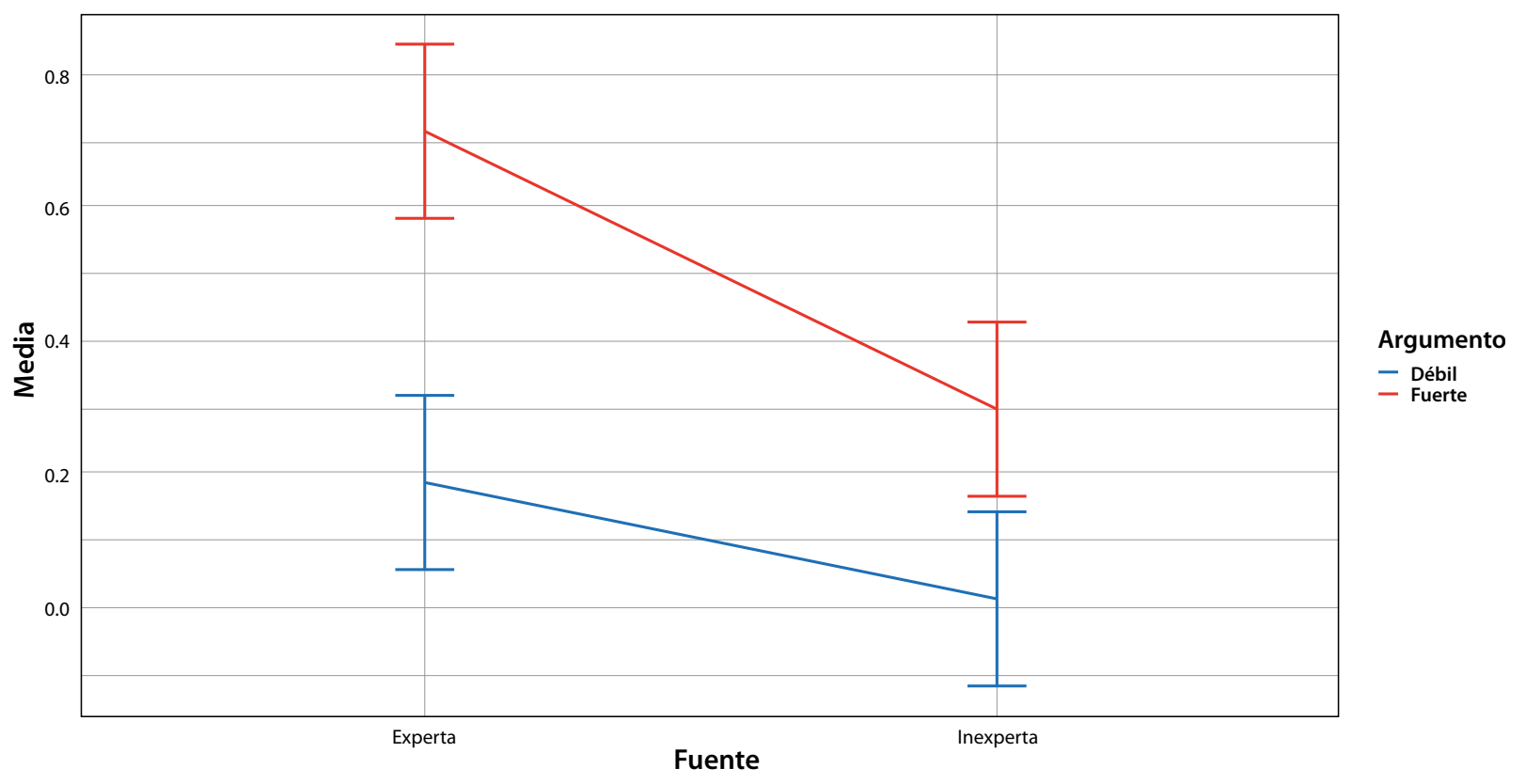


Figura 2.

Porcentaje de participantes según el tipo de respuesta

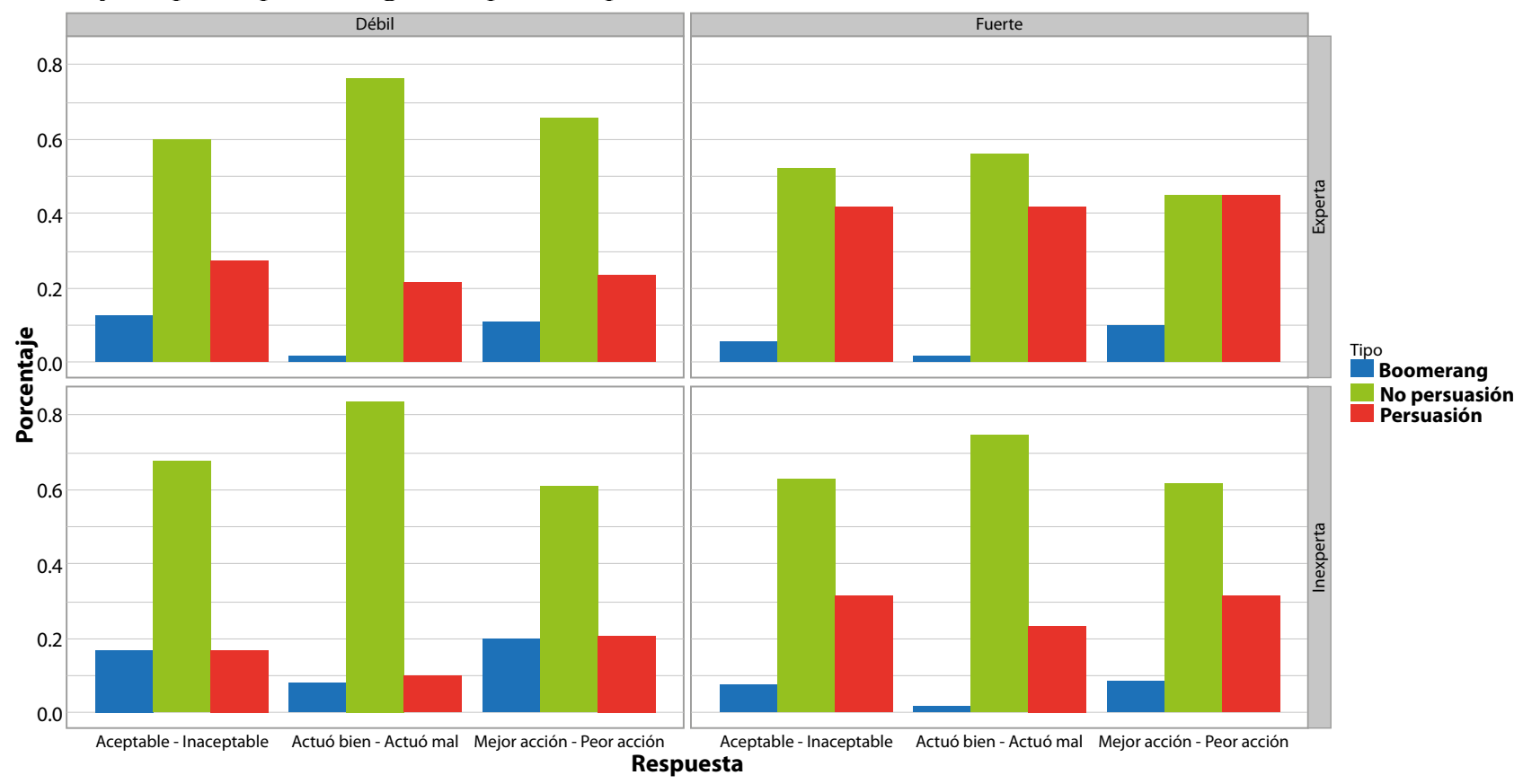

Argumento fuerte y fuente experta

A pesar de los bajos niveles de persuasión hallados, la prueba ANOVA permitió identificar un efecto principal de la calidad del argumento y la experticia de la fuente, a partir del cual se entiende que los argumentos fuertes producen más persuasión moral que los argumentos débiles, y que los mensajes proporcionados por una fuente experta generan más persuasión que los proporcionados por una inexperta. Los efectos de estos dos factores son consistentes con la TPD, en la cual se contempla que la persuasión moral se puede generar tanto por procesos automáticos — como los implicados en el cambio de juicios morales por efecto de la experticia de la fuente- como por procesos controlados - encargados de analizar la calidad de un argumento-. Para cada uno de estos dos efectos se puede proponer algunas explicaciones.

Con respecto a la influencia de la calidad de los argumentos, hay dos explicaciones complementarias que pueden dar cuenta de este efecto. La primera sugiere que el argumento contraactitudinal parece haber generado un conflicto cognitivo en los participantes, lo que llevó a la activación del sistema de procesamiento esforzado. En ese caso, es probable que el escrutinio del argumento haya generado persuasión en las personas que recibieron el argumento fuerte, y resistencia o efecto boomerang en quienes recibieron el argumento débil.
Esta explicación permite pensar dos aspectos en relación con el MIS y la TPD. Por una parte, Haidt (2001) plantea que la persuasión razonada - la persuasión en función de los argumentos - suele ocurrir sin necesidad de que se active el sistema de procesamiento esforzado, y que los argumentos persuasivos activan nuevas intuiciones y cambios en los juicios morales sin que haya razonamiento de por medio. $\mathrm{Si}$ en el presente estudio se hubiese dado esta situación, tanto los argumentos fuertes como los débiles habrían generado el mismo grado de persuasión, puesto que, desde esta perspectiva, cabría esperar que no sea necesario que los argumentos sean fuertes para activar intuiciones. En lugar de ello, se encontró que los argumentos fuertes fueron más persuasivos que los débiles, lo cual es consistente con la activación del sistema de procesamiento esforzado, cuya participación es necesaria para discriminar si un argumento es fuerte o débil. Adicionalmente, este hallazgo proporciona evidencia a favor de los planteamientos de Paxton y Greene (2010), quienes sostienen que diferentes formas de influencia social hacen que las personas consideren ciertos principios y obligaciones morales, y que anulen sus juicios automáticos. Los argumentos fuertes empleados en el estudio dan cuenta de ciertos principios morales: la justicia en el caso de los argumentos en contra, y el bienestar en el caso de los argumentos a favor. Estos argumentos parecen haber hecho que las personas cuestionaran sus juicios morales iniciales 
152

y cambiaran sus posturas por otras más consistentes con estos principios.

La segunda explicación complementa la anterior y apunta a la motivación, pues la presencia de una cuestión moral en un mensaje persuasivo parece hacer que, al igual que la implicación personal (Cancela et al., 2021), se incremente la motivación para procesar de manera sistemática la información. La influencia de la calidad de los argumentos que se halló en el estudio se podría atribuir a que el ingrediente moral del mensaje persuasivo aumentó la motivación y favoreció el escrutinio de los mensajes.

Por otra parte, con respecto al efecto de la experticia de la fuente, retomando las cinco formas en las que puede operar una señal heurística en la persuasión (Petty \& Briñol, 2006), es posible proponer tres explicaciones diferentes. En primer lugar, la experticia de la fuente pudo haber operado como una señal heurística que produjo en los participantes cierta respuesta intuitiva que los llevó a cambiar su juicio moral; no obstante, esta explicación no se ajustaría a todos los casos donde la experticia de la fuente impactó en la persuasión, pues esta forma de operar se produce cuando la motivación y la capacidad para procesar el mensaje persuasivo son insuficientes, y porque el efecto de la calidad de los argumentos del cual se habló antes sugiere todo lo contrario: que los participantes se sentían motivados y tenían la capacidad para procesar sistemáticamente la información.

En segundo lugar, es factible que los participantes hayan empleado una suerte de silogismo (Kruglanski, 2011) para inferir que si un juicio determinado procedía de un experto entonces debería ser válido. Este argumento extra, sumado al argumento fuerte del mensaje persuasivo, pudo haber producido más persuasión que cuando se presentó un argumento fuerte por una fuente inexperta o cuando una fuente experta dio un argumento débil.

$\mathrm{Y}$ en tercer lugar, la experticia de la fuente pudo haber sesgado de manera positiva la producción de pensamientos sobre el mensaje persuasivo. Según esta interpretación, la fuente experta influyó en el procesamiento de los mensajes, lo cual ocasionó que se tuviera una consideración más favorable de ellos. De este modo, el argumento fuerte hizo que se potenciara aún más la producción de pensamientos favorables, y con ello generó más persuasión que cuando el argumento fue débil. Por otra parte, la fuente inexperta pudo no haber producido un sesgo - ni positivo ni negativo-, pues si bien la descripción de la fuente inexperta daba cuenta de un hombre que no tenía experiencia en el tema, y no se mencionaba ningún aspecto negativo que produjera un sesgo. Ello explicaría por qué el grupo "argumento fuerte/fuente inexperta" no tuvo el incremento que la fuente experta dio al argumento fuerte en el grupo "argumento fuerte/fuente experta" (véase Figura 1).
Las demás formas de operar de las señales heurísticas no serían factibles en este caso, pues no es posible que la experticia de la fuente haya autovalidado la persuasión, dado que una variable afecta la confianza en los cambios de actitudes cuando es introducida después de la presentación del mensaje, y no antes, como ocurrió en este estudio. La explicación de la fuente experta como un factor que aumenta la motivación para el procesamiento sistemático del mensaje exige una interacción en la cual la fuente experta produzca persuasión, pero solo cuando esté acompañada del argumento fuerte, pues la persuasión con argumentos débiles no sería factible dada la motivación para escrutarlos. Como se mencionó en los resultados, esta interacción no fue significativa, y la fuente experta acompañada del argumento débil produjo persuasión, incluso más que cuando se presentó la fuente inexperta y el argumento débil (véase Figura 1).

\section{Convicciones morales y resistencia a la persuasión moral}

Sin quitar mérito a la anterior discusión, un dato que destaca es la resistencia a la persuasión que se encontró en el estudio. Como lo muestra la Figura 2, la mayoría de los participantes conservó su postura inicial sin importar la condición. Este aspecto parece ser congruente con trabajos anteriores (Ben-Nun Bloom \& Levitan, 2011) y con los planteamientos de la TICM (Skitka et al., 2021) que sugieren que las convicciones morales son inmunes a diferentes formas de influencia social. No obstante, la resistencia a la persuasión moral no se presentó de acuerdo con el grado en que los participantes consideraban que sus posiciones sobre el dilema estaban relacionadas con sus creencias y convicciones morales, como sugiere la TICM.

Para explicar esta contradicción, es necesario recordar que los juicios morales se refieren a evaluaciones de una acción o del carácter de una persona en función de su deber ser moral, mientras que las convicciones morales son aquellas actitudes que se perciben como un reflejo de las creencias morales. Así pues, de acuerdo con los resultados del estudio, se puede decir que no hace falta reconocer el nexo de una posición determinada con las creencias morales para presentar resistencia a la persuasión. Basta con que una postura trate sobre un asunto de tinte moral para que sea inmune contra las tentativas de persuasión.

Infiriendo más allá de los resultados obtenidos, se podría plantear que los juicios morales - dada su cualidad moralpresentan cierta inercia propia que los hace resistentes al cambio a través de la persuasión. De ser así, la mayoría de los participantes habría mantenido sus posturas debido a esta inercia inherente de sus juicios morales. Probablemente, los participantes presentaron un sesgo negativo para procesar los mensajes persuasivos (Petty \& Briñol, 2011), 
experimentaron disonancia cognitiva al encontrar información contradictoria con su posición (Wood, 2000), o bien presentaron un sentimiento de reactancia al percibir las tentativas de persuasión (Steindl et al., 2015), pero, en cualquiera de estos casos, conservaron sus posturas. Así, se puede afirmar que las tentativas de persuasión chocan contra la inercia propia de los juicios morales y de estos mecanismos. Solo bajo determinadas circunstancias - como cuando los argumentos son fuertes y los proporciona una fuente conocedora- pueden generar algún impacto en los juicios morales.

Finalmente, es importante mencionar que el presente estudio presenta tres limitaciones importantes que podrían ser corregidas en futuros estudios. En primer lugar, el efecto de la manipulación de la calidad del argumento fue limitado, como se pudo ver en su verificación, donde no hubo una diferencia tan amplia entre las valoraciones de los argumentos fuertes o débiles. Además, es claro que las personas no suelen estimar la fuerza de un argumento a partir de su validez formal. Futuras investigaciones podrían explorar nuevas formas de manipular esta variable, la cual, como se indicó en la introducción, siempre ha generado problemas en su manipulación (O'Keefe \& Jackson, 1995).

Una segunda limitación está relacionada con el desenlace del dilema moral empleado, pues este presenta una omisión - no delatar al desmovilizado — . Las omisiones y las acciones suelen ser juzgadas de manera diferente. Incluso, aunque presenten las mismas consecuencias, las acciones son juzgadas de manera más severa que las omisiones (Haidt \& Baron, 1996). Probablemente, el hecho de que el dilema moral empleado haya presentado una omisión en lugar de una acción afectó en alguna medida los juicios morales de los participantes y el cambio de sus posturas después del mensaje persuasivo. Futuros estudios podrían tener presente este tipo de sesgos a la hora de diseñar escenarios morales para estudiar la influencia social en temas de índole política en Colombia.

Una tercera limitación, que invita a tomar los hallazgos con cautela, está relacionada con la validez externa de los resultados. A menudo, los dilemas morales que suelen darse en la actualidad generan grandes polémicas en las que comúnmente hay muchos factores implicados que pueden afectar la susceptibilidad de las personas a cambiar de opinión. Si bien el estudio presentado permite mejorar nuestra comprensión de un evento particular de la actualidad colombiana, los materiales del experimento implican un dilema altamente contextualizado a partir del cual la posibilidad de generalización es limitada.

Teniendo en cuenta la utilidad de la TFM para pensar temas políticos (Graham et al., 2013), futuras investigaciones podrían explorar el moral reframing (Feinberg \& Willer,
2019) como una herramienta para estudiar la persuasión y facilitar la discusión en relación con diferentes coyunturas políticas y sociales, teniendo en cuenta los fundamentos morales a los cuales dan relevancia los colombianos de diferentes identidades políticas.

\section{Conclusiones}

En resumen, el presente trabajo hace un acercamiento al tema de la persuasión moral y, específicamente, explora el efecto de la calidad de los argumentos y la experticia de la fuente en el cambio de juicios morales por medio de la persuasión. En general, los resultados se ajustan a la TPD, en la cual se considera que la persuasión moral se puede producir tanto por una vía de procesamiento sistemático como por un tipo de procesamiento automático (Paxton \& Greene, 2010). La cualidad moral de los juicios morales parece coincidir con un procesamiento sistemático del mensaje persuasivo, a partir del cual se genera persuasión cuando se presentan argumentos fuertes. Por su parte, la experiencia y el conocimiento de la fuente parecen operar como una señal heurística, como un argumento extra, o como un sesgo positivo de los pensamientos sobre el mensaje, lo que genera, a su vez, más persuasión. Los efectos de estos dos factores pueden sumarse el uno al otro, lo cual ocasionaría que haya más persuasión cuando hay un argumento fuerte y una fuente experta que cuando se presenta solo uno de estos dos aspectos.

El presente trabajo proporciona ciertas claves acerca de las discusiones que se dan en torno a temas morales de gran controversia - como el proceso de paz en Colombia - para hacer que las personas sean más críticas al momento de considerar sus posiciones y la información que reciben. A diferencia de lo que sugiere la TICM (Skitka et al., 2021), las personas pueden modificar sus posiciones morales, y, en contraposición a lo que plantea el MIs (Haidt, 2012), dichos cambios pueden tener lugar con la participación de un tipo de procesamiento sistemático para analizar la calidad de los argumentos. Es necesario estudiar el tipo de factores que pueden favorecer la activación de este tipo de procesamiento en temas morales tan importantes como el proceso de paz en Colombia; sin embargo, los resultados también revelan que puede haber procesos automáticos implicados en estos cambios, en los que señales heurísticas como la experticia de la fuente pueden facilitar la persuasión. No obstante, esto último puede resultar en un arma de doble filo, dado que puede ser empleado tanto para bien como para mal. Frente a este riesgo, se puede educar a las personas acerca de esta clase de efectos para que, al momento de considerar un argumento, traten de evaluar si lo están valorando 
154

en función de su calidad o de la fuente de donde procede. Si bien esto es un ejercicio metacognitivo sumamente complejo, el reconocer los sesgos en los que es posible incurrir puede hacer que las personas estén más atentas a sus juicios y traten de evaluar de manera más imparcial la información (Paxton \& Greene, 2010).

\section{Referencias}

Aramovich, N., Lytle, B., \& Skitka, L. (2012). Opposing torture: Moral conviction and resistance to majority influence. Social Influence, 7(1). 21-34. https://doi.org/10.1080/1553 4510.2011.640199

Ben-Nun Bloom, P., \& Levitan, L. C. (2011). We're closer than I thought: Social network heterogeneity, morality, and political persuasion. Political Psychology, 32(4), 643-665. https://doi.org/10.1111/j.1467-9221.2011.00826.x

Bennett, W. L., \& Livingston, S. (2018). The disinformation order: Disruptive communication and the decline of democratic institutions. European journal of communication, 33(2), 122-139. https://doi.org/10.1177/0267323118760317

Briñol, P., \& Petty, R. (2009). Source factors in persuasion: A selfvalidation approach. European review of social psychology, 20(1), 49-96. https://doi.org/10.1080/10463280802643640

Buttrick, N., Moulder, R., \& Oishi, S. (2020). Historical Change in the Moral Foundations of Political Persuasion. Personality and Social Psychology Bulletin, 46(11), 15231537. https://doi.org/10.1177/0146167220907467

Cancela, A., Briñol, P., \& Petty, R. E. (2021). Hedonic vs. epistemic goals in processing persuasive communications: Revisiting the role of personal involvement. Motivation and Emotion, 45(3), 280-298. https://doi.org/10.1007/ s11031-021-09873-7

Carpenter, C. J. (2015). A meta-analysis of the ELM's argument quality processing type predictions. Human Communication Research, 41(4), 501-534. https://doi. org $/ 10.1111 /$ hcre. 12054

Chaiken, S., \& Ledgerwood, A. (2011). A theory of heuristic and systematic information processing. En P. A. Van Lange, A. W. Kruglanski y E. T. Higgins (Eds.), Handbook of theories of social psychology: Volume two (Vol. 1, pp. 246166.). SAGE publications.

Christensen, J., \& Gomila, A. (2012). Moral dilemmas in cognitive neuroscience of moral decision-making: A principled review. Neuroscience and Biobehavioral Reviews, 36, 12491264. https://doi.org/10.1016/j.neubiorev.2012.02.008

Clark, J. K., Evans, A. T., \& Wegener, D. T. (2011). Perceptions of source efficacy and persuasion: Multiple mechanisms for source effects on attitudes. European Journal of Social Psychology, 41(5), 596-607. https://doi.org/10.1002/ ejsp.787
DeBono, K. G., \& Harnish, R. J. (1988). Source expertise, source attractiveness, and the processing of persuasive information: A functional approach. Journal of Personality and social Psychology, 55(4), 541. https://doi. org/10.1037/0022-3514.55.4.541

Faul, F., Erdfelder, E., Buchner, A., \& Lang, A.-G. (2009). Statistical power analyses using G*Power 3.1: Tests for correlation and regression analyses. Behavior Research Methods, 41, 1149-1160. https://doi.org/10.3758/ BRM.41.4.1149

Feinberg, M., \& Willer, R. (2013). The moral roots of environmental attitudes. Psychological Science, 24(1), 56-62. https://doi.org/10.1177/0956797612449177

Feinberg, M., \& Willer, R. (2015). From gulf to bridge: When do moral arguments facilitate political influence? Personality and Social Psychology Bulletin, 41(12), 1665-1681. https:// doi.org/10.1177/0146167215607842

Feinberg, M., \& Willer, R. (2019). Moral reframing: A technique for effective and persuasive communication across political divides. Social and Personality Psychology Compass, 13(12), e12501. https://doi.org/10.1111/spc3.12501

Graham, J., Haidt, J., Koleva, S., Motyl, M., Iyer, R., Wojcik, S. P., \& Ditto, P. H. (2013). Moral foundations theory: The pragmatic validity of moral pluralism. Advances in experimental social psychology (vol. 47, pp. 55-130). Academic Press. https://doi.org/10.1016/B978-0-12-407236-7.00002-4

Graham, J., Haidt, J., \& Nosek, B. A. (2009). Liberals and conservatives rely on different sets of moral foundations. Journal of Personality and Social Psychology, 96(5), 1029. https://doi.org/10.1037/a0015141

Greene, J. (2013). Moral tribes: Emotion, reason, and the gap between $u$ and them. Penguin.

Haidt, J. (2001). The emotional dog and its rational tail: a social intuitionist approach to moral judgment. Psychological Review, 108(4), 814-834. https://doi. org/10.1037/0033-295X.108.4.814

Haidt, J. (2012). The righteous mind: Why good people are divided by politics and religion. Vintage.

Haidt, J., \& Baron, J. (1996). Social roles and the moral judgement of acts and omissions. European Journal of Social Psychology, 26(2), 201-218. https://doi. org/10.1002/(SICI)1099-0992(199603)26:2<201::AIDEJSP745>3.0.CO;2-J

Hornsey, M., Smith, J. R., \& Begg, D. (2007). Effects of norms among those with moral conviction: Counter-conformity emerges on intentions but not behaviors. Social Influence, 2(4), 244-268. https://doi.org/10.1080/15534510701476500

Kruglanski, A. W. (2011). Lay epistemic theory. En P. A. Van Lange, A. W. Kruglanski y E. T. Higgins (Eds.), Handbook of theories of social psychology: Volume two (vol. 1, pp. 201-223). SAGE publications.

Luttrell, A., Petty, R., Briñol, P., \& Wagner, B. (2016). Making it moral: Merely labeling an attitude as moral increases its 
strength. Journal of Experimental Social Psychology, 65, 82-93. https://doi.org/10.1016/j.jesp.2016.04.003

Moses, J. F., \& Gonzales, M. H. (2015). Strong candidate, nurturant candidate: Moral language in presidential television advertisements. Political Psychology, 36(4), 379-397. https://doi.org/10.1111/pops. 12160

O’Keefe, D. J., \& Jackson, S. (1995). Argument quality and persuasive effects: A review of current approaches. En S. Jackson (Ed.), Argumentation and values: Proceedings of the ninth Alta conference on argumentation (pp. 88-92). Speech Communication Association.

Paxton, J. M., \& Greene, J. D. (2010). Moral reasoning: Hints and allegations. Topics in cognitive science, 2(3), 511-527. https://doi.org/10.1111/j.1756-8765.2010.01096.x

Paxton, J. M., Ungar, L., \& Greene, J. D. (2012). Reflection and reasoning in moral judgment. Cognitive Science, 36(1), 163-177. https://doi.org/10.1111/j.1551-6709.2011.01210.x

Petty, R. E., \& Briñol, P. (2006). Understanding social judgment: Multiple systems and processes. Psychological Inquiry, 17(3), 217-223. https://www.jstor.org/stable/20447327

Petty, R. E., \& Briñol, P. (2011). The elaboration likelihood model. En P. A. Van Lange, A. W. Kruglanski y E. T. Higgins, (Eds), Handbook of theories of social psychology: Volume two (vol. 1, pp. 224-245.). SAGE publications.

Pornpitakpan, C. (2004). The persuasiveness of source credibility: A critical review of five decades' evidence. Journal of Applied Social Psychology, 34(2), 243-281. https://doi. org/10.1111/j.1559-1816.2004.tb02547.x

Redacción El Tiempo. (2 de octubre de 2016). Antioquia, Santanderes y Eje, regiones que dieron el triunfo al 'No'. El tiempo. https://www.eltiempo.com/archivo/documento/ CMS-16716975

Semana. (9 de febrero de 2016b). Plebiscito: polarización entre "fachos" y "castro-chavistas". Semana. https://www.semana.com/nacion/articulo/plebiscito-por-la-paz-entre-fachosy-castrochavistas/492268/

Semana. (25 de septiembre de 2016a). "Lo que he tratado es de abrirles los ojos a los colombianos": Alejandro Ordóñez. Semana. https://www.semana.com/nacion/articulo/alejandro-ordonez-habla-del-proceso-de-paz-el-gobierno-santosla-ideologia-de-genero-y-el-plebiscito/495287/

Skitka, L. J., Bauman, C. W., \& Lytle, B. L. (2009). Limits on legitimacy: moral and religious convictions as constraints on deference to authority. Journal of Personality and Social Psychology, 97(4), 567-578. https://doi.org/10.1037/ a0015998
Skitka, L. J., Hanson, B. E., Morgan, G. S., \& Wisneski, D. C. (2021). The psychology of moral conviction. Annual Review of Psychology, 72, 347-366. https://doi.org/10.1146/ annurev-psych-063020-030612

Steindl, C., Jonas, E., Sittenthaler, S., Traut-Mattausch, E., \& Greenberg, J. (2015). Understanding psychological reactance. Zeitschrift für Psychologie, 223(4), 205-214. https://doi. org/10.1027/2151-2604/a000222

Tormala, Z. L., \& Briñol, P. (2015). Attitude change and persuasion: Past, present, and future directions. En M. I. Norton, D. D. Rucker y C. Lamberton(Eds.), The Cambridge Handbook of consumer psychology (pp 29-64). Cambridge University Press. https://doi.org/10.1017/CBO9781107706552.002

Van Lange, P. A., Kruglanski, A. W., \& Higgins, E. T. (2011). Handbook of theories of social psychology: Volume two (vol. 2). SAGE publications.

Wood, W. (2000). Attitude change: Persuasion and social influence. Annual review of psychology, 51(1), 539-570. https://doi.org/10.1146/annurev.psych.51.1.539

\section{Anexos}

Anexo 1. Formulario de Google https://goo.gl/forms/ygVCb4oWNpMYkk8j2

Anexo 2. Enlaces de vídeos empleados en el estudio

Dilema moral del personero: https://www.youtube.com/ watch?v=ne_DsILeiqM \& $\mathrm{t}=3 \mathrm{~s}$

Descripción del perfil de la fuente inexperta: https:// www.youtube.com/watch?v=B_KT-FNMb3U

Descripción del perfil de la fuente experta: https://www. youtube.com/watch? $\mathrm{v}=\mathrm{fpf} 32 \mathrm{qDDOuQ}$

Argumento fuerte en contra: https://www.youtube.com/ watch? $=$ NuHW_L716QA

Argumento fuerte a favor: https://www.youtube.com/ watch? $v=19 \mathrm{r} 2 \mathrm{Q} 7 \mathrm{~g} 3 \mathrm{RaE}$

Argumento débil a favor: https://www.youtube.com/ watch? $\mathrm{v}=$ NnnwbtsyGZ8

Argumento débil en contra: https://www.youtube.com/ watch? $\mathrm{v}=$ PuiwSHuWiPU 